\title{
The Kinematic Image of 2R Dyads and Exact Synthesis of 5R Linkages
}

\author{
Tudor-Dan Rad and Hans-Peter Schröcker \\ Unit Geometry and CAD \\ University of Innsbruck, Austria
}

September 14, 2018

\begin{abstract}
We characterise the kinematic image of the constraint variety of a $2 \mathrm{R}$ dyad as a regular ruled quadric in a three-space that contains a "null quadrilateral". Three prescribed poses determine, in general, two such quadrics. This allows us to modify a recent algorithm for the synthesis of $6 \mathrm{R}$ linkages in such a way that two consecutive revolute axes coincide, thus producing a 5R linkage. Using the classical geometry of twisted cubics on a quadric, we explain some of the peculiar properties of the the resulting synthesis procedure for $5 \mathrm{R}$ linkages.
\end{abstract}

Keywords: 2R dyad, kinematic map, 5R linkage, Goldberg linkage, linkage synthesis, dual quaternions

MSC 2010: 70B15 (primary); 70B10, 12D05, 51N15

\section{Introduction}

Given three poses of a rigid body, there exists, in general, a unique closed kinematic loop of four revolute joints with one degree of freedom, a so-called Bennett or 4R linkage, one of whose links visits the three poses. Suh (1969) solved this synthesis problem by constructing two $2 \mathrm{R}$ dyads to the three given poses and proving that they can be combined to form a Bennett linkage. The three pose synthesis problem for $4 \mathrm{R}$ linkages was re-visited by Brunnthaler et al. (2005) in a dual quaternion setting. A generalisation of their approach led to a factorisation theory for "motion polynomials" (Hegedüs et al., 2013a). It was used by Hegedüs et al. (2014) to synthesise spatial 6R linkages - closed kinematic chains with six revolute joints - to four prescribed poses in general position. More precisely, the four poses determine two families of (not necessary real) rational coupler motions, each giving rise to nine families of overconstrained $6 \mathrm{R}$ linkages. It should also be mentioned that the construction is only capable of producing a special type of $6 \mathrm{R}$ linkages. 
In this paper we intend to close the gap in this sequence of exact factorisation based synthesis procedures for closed loop linkages and present a construction of 5R linkages (Goldberg linkages) to "more than three but less than four poses". Our synthesis algorithm is based on a characterisation of the image of open 2R chains under Study's kinematic mapping and the observation that the coupler motion of a $5 \mathrm{R}$ linkage is also parameterised by a cubic motion polynomial (see Hegedüs et al., 2013b). This allows to treat $5 \mathrm{R}$ synthesis as a special case of above mentioned synthesis of $6 \mathrm{R}$ linkages.

We continue this text with a quick introduction to dual quaternions and kinematics in Section 2. In Section 3 we provide a geometric characterisation for the kinematic image of a $2 \mathrm{R}$ dyad. While necessary properties can be derived easily, the proof of their sufficiency requires more work and comprises the main part of this text. The synthesis of $5 \mathrm{R}$ linkage in Section 4 is then merely a corollary to previous results. Note however, that it is not obvious how to use the additional degrees of freedom in exact $5 \mathrm{R}$ synthesis.

\section{Preliminaries}

This article's scene is the projectivised dual quaternion model of spatial kinematics. Here, we give a very brief introduction to this model for the purpose of settling our notation. More details will be introduced in the text as needed. For more thorough introductions to dual quaternions and there relations to kinematics we refer to (Klawitter, 2015, Section 3) or (Selig, 2005, Section 11). Familiarity with Hegedüs et al. (2013a) is recommended too.

The dual quaternions, denoted by $\mathbb{D H}$, form an associative algebra in $\mathbb{R}^{8}$ where multiplication of the base elements $1, \mathbf{i}, \mathbf{j}, \mathbf{k}, \varepsilon, \varepsilon \mathbf{i}, \varepsilon \mathbf{k}$, and $\varepsilon \mathbf{k}$ is defined by the rules

$$
\mathbf{i}^{2}=\mathbf{j}^{2}=\mathbf{k}^{2}=\mathbf{i j k}=-1, \quad \varepsilon^{2}=0, \quad \mathbf{i} \varepsilon=\varepsilon \mathbf{i}, \quad \mathbf{j} \varepsilon=\varepsilon \mathbf{j}, \quad \mathbf{k} \varepsilon=\varepsilon \mathbf{k} .
$$

An element $q \in \mathbb{D} \mathbb{H}$ may be written as $q=p+\varepsilon d$ with quaternions $p, d \in \mathbb{H}:=\langle 1, \mathbf{i}, \mathbf{j}, \mathbf{k}\rangle$ (angled brackets denote linear span). In this case the quaternions $p$ and $d$ are referred to as primal and dual part of $q$, respectively. The conjugate dual quaternion is $\bar{q}=\bar{p}+\varepsilon \bar{d}$ and conjugation of quaternions is done by multiplying the coefficients of $\mathbf{i}, \mathbf{j}$, and $\mathbf{k}$ with -1 . It satisfies the rule $\overline{q r}=\bar{r} \bar{q}$ for any $q, r \in \mathbb{D} \mathbb{H}$. The dual quaternion norm is defined as $\|q\|=q \bar{q}$. We readily verify that it is a dual number, that is, an element of $\mathbb{D}:=\langle 1, \varepsilon\rangle$.

We identify linearly dependent non-zero dual quaternions and thus arrive at the projective space $P^{7}=P\left(\mathbb{R}^{8}\right)$. Writing $[q]$ for the point in $P^{7}$ that is represented by $q \in \mathbb{D} \mathbb{H}$, the Study quadric is defined as $\mathcal{S}:=\left\{[q] \in P^{7}:\|q\| \in \mathbb{R}\right\}$. With $q=p+\varepsilon d$, the algebraic condition for $[q] \in \mathcal{S}$ is $p \bar{d}+d \bar{p}=0$.

Identifying $P^{3}$ with the projective subspace generated by $\langle 1, \varepsilon \mathbf{i}, \varepsilon \mathbf{j}, \varepsilon \mathbf{k}\rangle$, a point $[q]=$ $[p+\varepsilon d] \in \mathcal{S}$ with non-zero primal part acts on $[x] \in P^{3}$ via

$$
[x] \mapsto[(p+\varepsilon d) x(\bar{p}-\varepsilon \bar{d})] .
$$

The map (1) is the projective extension of a rigid body displacement in $\mathbb{R}^{3}$. Composition of displacements corresponds to dual quaternion multiplication. This isomorphism between $\mathrm{SE}(3)$, the group of rigid body displacements, and the projectivisation of the 
group of dual quaternions of real norm and non-zero primal part provides a rich and solid algebraic and geometric basis for investigations in kinematics.

\section{A characterisation of $2 R$ spaces}

We complement the Study quadric by the quadric $\mathcal{N}=\{[q]:\|q\| \in \varepsilon \mathbb{R}\}$ of points represented by dual quaternions whose norm has vanishing primal part. It is a quadric of rank four. The set $E=\{[q]: q \in \varepsilon \mathbb{H}\}$ of its singular points is a projective space of dimension three and it is contained in the Study quadric. Only the points of $E$ are real points of $\mathcal{N}$ whence we are led to consider the complex extension $P\left(\mathbb{C}^{8}\right)$ of $P^{7}$. We call $\mathcal{N}$ the null cone and refer to the set $E$ of its singular points as the exceptional three space.

Given two non coplanar lines $\ell_{1}, \ell_{2}$ in Euclidean three space, we consider the set of all displacements obtained as composition of a rotation around $\ell_{2}$, followed by a rotation about $\ell_{1}$. Its kinematic image is known to lie in a three space (Selig, 2005, Section 11.4) which we call a $2 R$ space. By construction, a $2 \mathrm{R}$ space contains the point [1] (the identity displacement) and intersects $\mathcal{S}$ in a doubly ruled regular quadric. The rulings are obtained by varying one revolute angle and fixing the other. In order to characterise $2 \mathrm{R}$ spaces among all three-dimensional subspaces of $P^{7}$ with these properties, we introduce the notion of null quadrilaterals. These are spatial quadrilaterals contained in both, $\mathcal{S}$ and $\mathcal{N}$. We call their edges null lines.

Theorem 1. A three space $P \subset P^{7}$ with $[1] \in P$ is a $2 R$ space if and only if it

- intersects the Study quadric in a regular ruled quadric $\mathcal{Q}$,

- does not intersect the exceptional three space E, and

- contains a null quadrilateral.

The first and second item in Theorem 1 exclude exceptional cases with coplanar, complex, or "infinite" revolute axes, the latter corresponding to prismatic joints. The important point is existence of a null quadrilateral. We split the proof of Theorem 1 into a series of lemmas. It will be finished by the end of this section.

Lemma 1. The straight line $[x] \vee[y]$ is contained in $\mathcal{S} \cap \mathcal{N}$ if and only if $x \bar{x}=y \bar{y}=$ $x \bar{y}+y \bar{x}=0$.

We omit the straightforward computational proof but note that the left-hand sides of each of the three conditions in Lemma 1 are dual numbers. Hence, they give just six independent conditions on the real coefficients of $x$ and $y$.

Lemma 2. The conditions of Theorem 1 are necessary for $2 R$ spaces.

Proof. The constraint variety of a $2 \mathrm{R}$ chain can be parameterised as

$$
R\left(t_{1}, t_{2}\right)=\left(t_{1}-h_{1}\right)\left(t_{2}-h_{2}\right)
$$


with two dual quaternions $h_{1}, h_{2}$ that satisfy $h_{1} \overline{h_{1}}=h_{2} \overline{h_{2}}=1, h_{1}+\overline{h_{1}}=h_{2}+\overline{h_{2}}=0$ and $h_{1} h_{2} \neq h_{2} h_{1}$. The first condition ensures that the dual quaternions are suitably normalised and $\left[h_{1}\right],\left[h_{2}\right] \in \mathcal{S}$. The second condition means that $h_{1}$ and $h_{2}$ describe half-turns (rotations through an angle of $\pi$ ). The third condition ensures that the axes of these half turns are different. Equation (2) describes a composition of two rotations for all values of $t_{1}, t_{2}$ in $\mathbb{R} \cup\{\infty\}$ with $\infty$ corresponding to zero rotation angle. Even if their kinematic meaning is unclear, we also allow complex parameter values. Expanding (2) yields

$$
R\left(t_{1}, t_{2}\right)=t_{1} t_{2}-t_{1} h_{2}-t_{2} h_{1}+h_{1} h_{2} .
$$

We see that the kinematic image of the $2 \mathrm{R}$ dyad lies in the three space spanned by [1], $\left[h_{1}\right],\left[h_{2}\right],\left[h_{1} h_{2}\right]$. In a suitable projective reference with these points as base points, the surface parameterised by (2) is the quadric with equation $x_{0} x_{3}-x_{1} x_{2}=0$ which is indeed regular and ruled.

The intersection of $P$ with the exceptional three space $E$ is non empty if and only if the primal part of $R\left(t_{1}, t_{2}\right)$ vanishes for certain parameter values $t_{1}, t_{2}$. This can only happen if the primal parts of $h_{1}$ and $h_{2}$ are linearly dependent over $\mathbb{R}$ but then the revolute axes are parallel and $P$, contrary to our assumption, is contained in $\mathcal{S}$. The other possibility for $P \subset \mathcal{S}$, intersecting revolute axes, has been excluded as well. Clearly, $P$ is not contained in the null cone $\mathcal{N}$ either. We claim that the intersection of $P$ and $\mathcal{N}$ consists of the four lines given by $t_{1}= \pm \mathrm{i}, t_{2}= \pm \mathrm{i}$. Indeed, they are null lines. Take for example $x=\left(\mathrm{i}-h_{1}\right)\left(t_{2}-h_{2}\right)$ and, in view of Lemma 1 , compute

$$
\begin{aligned}
x \bar{x} & =\left(\mathrm{i}-h_{1}\right)\left(t_{2}-h_{2}\right)\left(t_{2}-\overline{h_{2}}\right)\left(\mathrm{i}-\overline{h_{1}}\right)=\left(t_{2}-h_{2}\right)\left(t_{2}-\overline{h_{2}}\right)\left(\mathrm{i}-h_{1}\right)\left(\mathrm{i}-\overline{h_{1}}\right) \\
& =\left(t_{2}-h_{2}\right)\left(t_{2}-\overline{h_{2}}\right)\left(-\mathrm{i}^{2}-\mathrm{i}\left(h_{1}+\overline{h_{1}}\right)+h_{1} \overline{h_{1}}\right)=\left(t_{2}-h_{2}\right)\left(t_{2}-\overline{h_{2}}\right)(-1-0+1)=0 .
\end{aligned}
$$

This derivation uses the fact that $\left(t_{2}-h_{2}\right)\left(t_{2}-\overline{h_{2}}\right)$ is a real number and thus commutes with all other factors. The cases $t_{1}=-\mathrm{i}, t_{2}= \pm \mathrm{i}$ are similar so that we have verified all conditions of Theorem 1.

The proof of sufficiency is more involved. We need two additional lemmas from projective geometry which are formulated and proved in Appendix A.

Lemma 3. A three space $P$ that satisfies all conditions of Theorem 1 is a $2 R$ space.

Proof. Denote the vertices of the null quadrilateral (in that order) by $\left[u_{1}\right],\left[v_{1}\right],\left[u_{2}\right]$, and $\left[v_{2}\right]$. Intersecting the Study null quadrilateral with the tangent hyperplane of $\mathcal{S}$ at [1] yields points

$$
\left[m_{1}\right] \in\left[u_{1}\right] \vee\left[v_{1}\right], \quad\left[n_{1}\right] \in\left[v_{1}\right] \vee\left[u_{2}\right], \quad\left[m_{2}\right] \in\left[u_{2}\right] \vee\left[v_{2}\right], \quad\left[n_{2}\right] \in\left[v_{2}\right] \vee\left[u_{1}\right]
$$

of a planar and non-degenerate quadrilateral (Figure 1). The joins $\left[m_{1}\right] \vee\left[m_{2}\right]$ and $\left[n_{1}\right] \vee\left[n_{2}\right]$ are real lines and their real points correspond to rotations about fixed axes. Pick real rotation quaternions $\left[h_{1}\right] \in\left[m_{1}\right] \vee\left[m_{2}\right]$ and $\left[h_{2}\right] \in\left[n_{1}\right] \vee\left[n_{2}\right]$. The axes of these rotations are the only candidates for our $2 \mathrm{R}$ dyad. Hence, we have to show that either $\left[h_{1} h_{2}\right] \in P$ or $\left[h_{2} h_{1}\right] \in P$. It is easy to see that this is the case if and only if 


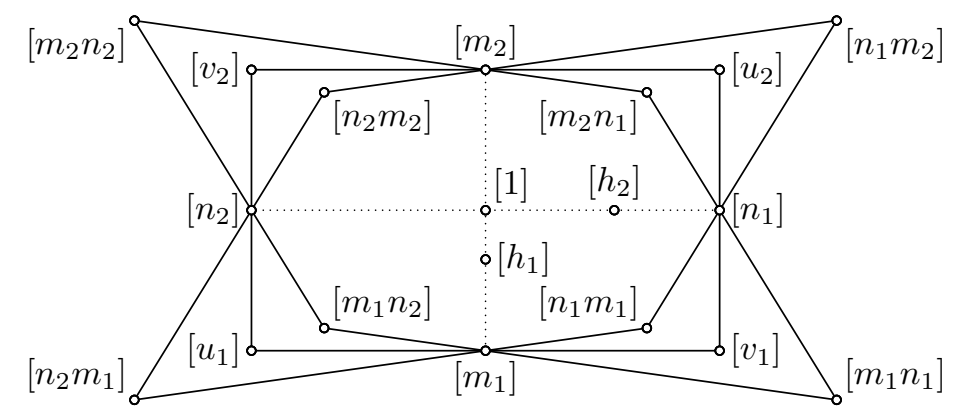

Figure 1: Null lines and null quadrilaterals in the proof of Lemma 3.

either $\left[m_{1} n_{1}\right] \in P$ or $\left[n_{1} m_{1}\right] \in P$. In fact, we will even show that one of these products equals $v_{1}$.

We claim that both $M_{1}:=\left[m_{1}\right] \vee\left[m_{1} n_{1}\right]$ and $M_{2}:=\left[m_{1}\right] \vee\left[n_{1} m_{1}\right]$ are null lines. In order to show this, we have to verify the conditions of Lemma 1 :

$$
\begin{gathered}
\left(m_{1} n_{1}\right) \overline{\left(m_{1} n_{1}\right)}=m_{1}(\underbrace{n_{1} \overline{n_{1}}}_{=0}) \overline{m_{1}}=0, \quad\left(n_{1} m_{1}\right) \overline{\left(n_{1} m_{1}\right)}=n_{1}(\underbrace{m_{1} \overline{m_{1}}}_{=0}) \overline{n_{1}}=0, \\
m_{1} \overline{\left(m_{1} n_{1}\right)}+\left(m_{1} n_{1}\right) \overline{m_{1}}=m_{1}(\underbrace{n_{1}+\overline{n_{1}}}_{\in \mathbb{C}}) \overline{m_{1}}=\left(n_{1}+\overline{n_{1}}\right)(\underbrace{m_{1} \overline{m_{1}}}_{=0})=0, \\
m_{1} \overline{\left(n_{1} m_{1}\right)}+\left(n_{1} m_{1}\right) \overline{m_{1}}=(\underbrace{m_{1} \overline{m_{1}}}_{=0}) \overline{n_{1}}+n_{1}(\underbrace{m_{1} \overline{m_{1}}}_{=0})=0 .
\end{gathered}
$$

Similarly, we see that also $N_{1}:=\left[n_{1}\right] \vee\left[m_{1} n_{1}\right]$ and $N_{2}:=\left[n_{1}\right] \vee\left[n_{1} m_{1}\right]$ are null lines. Thus, we are in the situation depicted in Figure 1 where we have three null quadrilaterals with respective vertices

$$
\left[u_{1}\right],\left[v_{1}\right],\left[u_{2}\right],\left[v_{2}\right] ; \quad\left[m_{1} n_{1}\right],\left[m_{2} n_{1}\right],\left[m_{2} n_{2}\right],\left[m_{1} n_{2}\right] ; \quad\left[n_{1} m_{1}\right],\left[n_{1} m_{2}\right],\left[n_{2} m_{2}\right],\left[n_{2} m_{1}\right] .
$$

The second and third quadrilateral are different because $m_{1}$ and $n_{1}$ do not commute (otherwise they would lie on the same line through [1] which contradicts the regularity of the quadric $\mathcal{Q}:=P \cap \mathcal{S}$ ). Our proof will be finished as soon as we have shown that the first and the second or the first and the third quadrilateral are equal. For this, it is sufficient to show that $\left[v_{1}\right]=\left[m_{1} n_{1}\right]$ or $\left[v_{1}\right]=\left[n_{1} m_{1}\right]$.

At first, we argue that the primal part of $\left[v_{1}\right]$ equals the primal part of $\left[m_{1} n_{1}\right]$ or of $\left[n_{1} m_{1}\right]$. Because $P$ does not intersect $E$, the projection on the primal part is a regular projectivity $P \rightarrow[\mathbb{H}]$ with centre $E$. We denote projected objects by a prime, that is, we write $u_{1}^{\prime}, v_{1}^{\prime}, m_{1}^{\prime}$ etc., for the primal parts of $u_{1}, v_{1}, m_{1}$ etc. The quadric $\mathcal{Q}$ is regular and ruled and so is its primal projection $\mathcal{Q}^{\prime}$. Hence, the point $\left[m_{1}^{\prime}\right] \in \mathcal{Q}^{\prime}$ is incident with precisely two lines contained in $\mathcal{Q}^{\prime}$, say $M_{1}^{\prime}$ and $M_{2}^{\prime}$. But $\left[m_{1}^{\prime}\right] \vee\left[v_{1}^{\prime}\right]$ is contained in $\mathcal{Q}^{\prime}$. Hence $\left[v_{1}^{\prime}\right] \in M_{1}^{\prime}$ or $\left[v_{1}^{\prime}\right] \in M_{2}^{\prime}$. Similarly, $\left[v_{1}^{\prime}\right]$ is also incident with one of the two lines contained in $\mathcal{Q}^{\prime}$ and incident with $\left[n_{1}^{\prime}\right]$. Thus, $\left[v_{1}^{\prime}\right]=\left[m_{1}^{\prime} n_{1}^{\prime}\right]$ or $\left[v_{1}^{\prime}\right]=\left[n_{1}^{\prime} m_{1}^{\prime}\right]$.

Now we have to lift this result to the dual part and show that $\left[v_{1}\right]=\left[m_{1} n_{1}\right]$ or $\left[v_{1}\right]=\left[n_{1} m_{1}\right]$. The alternative being similar, we assume $\left[v_{1}^{\prime}\right]=\left[m_{1}^{\prime} n_{1}^{\prime}\right]$. This means 
that we have two null quadrilaterals, one with vertices $\left[u_{1}\right],\left[v_{1}\right],\left[u_{2}\right],\left[v_{2}\right]$ and one with vertices $\left[m_{1} n_{1}\right],\left[m_{2} n_{1}\right],\left[m_{2} n_{2}\right],\left[m_{1} n_{2}\right]$ such that their primal projections are equal and corresponding sides intersect in the vertices $\left[m_{1}\right],\left[n_{1}\right],\left[m_{2}\right],\left[n_{2}\right]$ of a planar quadrilateral. But then Lemma 5 in Appendix A asserts their equality and $\left[v_{1}\right]=\left[n_{1} m_{1}\right]$ follows.

\section{Synthesis procedures}

In this section we discuss the exact synthesis of 5R linkages, based on our geometric characterisation of $2 \mathrm{R}$ spaces. The attentive reader will note that we neither provide a synthesis algorithm nor a concrete example. The reason for this (and one of the main points of this paper) is that the synthesis of $5 \mathrm{R}$ linkages can be treated as special case of a recently introduced synthesis procedure for $6 \mathrm{R}$ linkages (Hegedüs et al., 2014). In this context, $2 \mathrm{R}$ spaces play a crucial role.

At first, we re-prove a classical result of Suh (1969) on the synthesis of $2 \mathrm{R}$ dyads to three poses. Its proof is just a variant of our computations in the proof of Theorem 1 .

Theorem 2. Three poses $\left[p_{0}\right]=[1],\left[p_{1}\right],\left[p_{2}\right] \in \mathcal{S}$ that span a plane that is not tangent to $\mathcal{S}$ and does not intersect $E$ are incident with precisely two $2 R$ spaces.

Proof. By Theorem 1, the sought $2 \mathrm{R}$ space $P$ contains a null quadrilateral. We compute its vertices $\left[u_{1}\right],\left[v_{1}\right],\left[u_{2}\right],\left[v_{2}\right]$ as follows. The plane $\left[p_{0}\right] \vee\left[p_{1}\right] \vee\left[p_{2}\right]$ and the Study quadric $\mathcal{S}$ have a regular conic $C$ in common. This conic intersects the null cone in two pairs $m_{1}, m_{2}$, and $n_{1}, n_{2}$ of conjugate complex points that can be computed from the roots of a univariate quartic polynomial. Again, we denote projection on the primal part by a prime. We can determine the primal part $u_{1}^{\prime}$ of $u_{1}$ by solving the system $u_{1}^{\prime} \overline{u_{1}^{\prime}}=u_{1}^{\prime} \overline{m_{1}^{\prime}}+m_{1}^{\prime} \overline{u_{1}^{\prime}}=u_{1}^{\prime} \overline{n_{2}^{\prime}}+u_{1}^{\prime} \overline{n_{2}^{\prime}}=0$. It consists of two linear and one quadratic equation. Hence, there are precisely two solutions in projective sense. Picking one of them, the primal parts of the remaining vertices can be computed unambiguously: Write, for example, $v_{1}^{\prime}=u_{1}^{\prime}+\lambda m_{1}^{\prime}$ and solve $v_{1}^{\prime} \overline{n_{1}^{\prime}}+n_{1}^{\prime} \overline{v_{1}^{\prime}}=0$ for $\lambda$. Finally, from $\left[u_{1}^{\prime}\right],\left[v_{1}^{\prime}\right]$, $\left[u_{2}^{\prime}\right],\left[v_{2}^{\prime}\right]$ the points $\left[u_{1}\right],\left[v_{1}\right],\left[u_{2}\right],\left[v_{2}\right]$ can be uniquely recovered as in the proof of Lemma 5.

Remark 1. The two solutions in Theorem 2 both contain the conic $C$. This conic is the kinematic image of the coupler motion of a Bennett linkage (Hamann, 2011). Thus, as already mentioned by Suh (1969), the two $2 \mathrm{R}$ dyads of Theorem 2 can be combined to form a Bennett linkage. Moreover, the computation of Lemma 3 shows that the two $2 \mathrm{R}$ spaces are $[1] \vee\left[h_{1}\right] \vee\left[h_{2}\right] \vee\left[h_{1} h_{2}\right]$ and $[1] \vee\left[h_{1}\right] \vee\left[h_{2}\right] \vee\left[h_{2} h_{1}\right]$ with suitable rotation quaternions $h_{1}, h_{2}$. In other words, they differ only by the ordering of their axes. This shows that the figures formed by opposite axes in a Bennett linkage are congruent.

In a $5 \mathrm{R}$ linkage there are two possibilities to assign a coupler to a fixed base. The two choices can be distinguished by the degree of their relative motion with respect to the base. As has been shown recently by (Hegedüs et al., 2013b, Theorem 6), the degrees of relative motions between non-adjacent links in the dual quaternion model of rigid body kinematics are two and three, respectively. In order to gain additional degrees of 
freedoms over the synthesis of Bennett linkages, it is mandatory to use the link to the degree three motion as coupler. In this sense, we can say that the coupler motion of a 5R linkage admits a rational cubic parameterisation (a cubic motion polynomial) in the dual quaternion model of SE(3). However, not every twisted cubic in the Study quadric gives rise to a $5 \mathrm{R}$ linkage. The necessary and sufficient condition is that the coupler motion can also be generated by a $2 \mathrm{R}$ dyad, that is, the twisted cubic lies in a $2 \mathrm{R}$ space.

Using the factorisation theory for rational motions (Hegedüs et al., 2013a), a generic cubic motion polynomial $C$ can be written, in six different ways, as $C=\left(t-h_{1}\right)(t-$ $\left.h_{2}\right)\left(t-h_{3}\right)$ with linear motion polynomials $t-h_{i}, i \in\{1,2,3\}$, that parameterise rotations about fixed axes. Suitable combinations of such factorisations give an overconstrained 6R linkage whose coupler follows the prescribed cubic motion. For details on how to compute cubic interpolants on quadrics, how to factor the resulting motions and how to pick factorisations suitable for linkage synthesis we refer to Hegedüs et al. (2014). We just highlight the major differences to the case of $5 \mathrm{R}$ linkage synthesis.

By a fundamental property of the underlying theory, the factorisations of a cubic motion polynomial are, at least in generic situations, in bijection with the permutations of the real quadratic factors of $C \bar{C}$ (Hegedüs et al., 2013a). Pairings for "admissible" kinematic chains correspond to permutations with different factors at the beginning or at the end. In the case of $5 \mathrm{R}$ synthesis, the situation is special. At least one factorisation is of the shape $C=\left(t-h_{1}\right)\left(t-h_{2}\right)\left(t-h_{3}\right)$ where either the axes of $h_{1}$ and $h_{2}$ or of $h_{2}$ and $h_{3}$ are identical. Lets assume the first case. Then $h_{1}$ and $h_{2}$ commute so that $C=\left(t-h_{2}\right)\left(t-h_{1}\right)\left(t-h_{3}\right)$ is another factorisation, different in algebraic sense but identical in kinematic sense. It can be paired with just two of the remaining four factorisations to form a closed 5R linkage. If $h_{2}$ and $h_{3}$ have identical axis, above discussion can be repeated with $\bar{C}=\left(t-\overline{h_{3}}\right)\left(t-\overline{h_{2}}\right)\left(t-\overline{h_{1}}\right)$ instead of $C$.

For a more detailed discussion of some properties of $5 \mathrm{R}$ linkages in this context, we need some results of twisted cubics that can be found in classical texts (for example Salmon, 1882; Cayley, 1885). The intersection of a 2R space with the Study quadric is a regular ruled quadric $\mathcal{Q}$. It carries two families of rulings. One family contains a ruling corresponding, via Study's kinematic mapping, to all rotations about the axis at the base of the $2 \mathrm{R}$ dyad, the other family contains a ruling corresponding to the moving revolute axes. We may accordingly speak of a "first" and a "second" family of rulings. The twisted cubics on $\mathcal{Q}$ can be partitioned into two classes: Members of the first class intersect every ruling of the first family in two and every ruling of the second family in just one point. For cubics of second class, the situation is just the other way round. Finally, four points on $\mathcal{Q}$ determine two one-parametric families of twisted cubics, one of first class and one of second. A member of each family is uniquely determined by the choice of one further point on a ruling through one of the four given points.

These considerations clearly show that four poses in general position cannot be reached by a $5 \mathrm{R}$ linkage unless their kinematic images span a $2 \mathrm{R}$ space. On the other hand, three poses in general position already determine, by Theorem 2 , two $2 \mathrm{R}$ spaces but also a Bennett linkage. By above discussion, we have three degrees of freedom to find a cubic curve through three quadric points. In other words, the number of free degrees in $5 \mathrm{R}$ linkage synthesis exceed that of $4 \mathrm{R}$ linkages by three. Unfortunately, only restricted use 
can be made of these. It is, for example, not possible to arbitrarily prescribe one further orientation: While there is a dual quaternion with prescribed primal part (orientation) in each $2 \mathrm{R}$ space, it will, in general, not lie on the Study quadric. An additional prescribed position is prevented for similar reasons.

Finally, also the appearance of two types of factorisations with commuting factors on the left $\left(h_{1}, h_{2}\right)$ or on the right $\left(h_{2}, h_{3}\right)$ can be explained by the two families of twisted cubics on $\mathcal{Q}$. If the commuting factors are on the left, $C_{0}(t)=\left(t-h_{1}\right)\left(t-h_{2}\right)$ is a quadratic parameterisation of the line $[1] \vee\left[h_{1}\right]$ and every point on that line corresponds to two parameter values. In particular, there exist a second parameter value $t_{0}$ (besides $\infty)$ such that $\left[C_{0}\left(t_{0}\right)\right]=[1]$. But then $C\left(t_{0}\right)$ yields a second curve point (besides [1]) on $[1] \vee\left[h_{3}\right]$, that is, the cubic belongs to the second family. In similar manner we see that the cubic belongs to the first family, if $h_{2}$ and $h_{3}$ commute.

\section{Conclusion}

We characterised the kinematic image of $2 \mathrm{R}$ dyads and used it to specialise a recently developed synthesis procedure for $6 \mathrm{R}$ linkages in such a way that it yields $5 \mathrm{R}$ linkages. Even if sufficient degrees of freedom are available, it is not possible in general to find a $5 \mathrm{R}$ linkage that visits three and a half poses (three poses plus one position or one orientation) because of geometric obstructions. Thus, a natural selection of interpolation data for the envisaged coupler motion is not entirely clear. Hopefully, future research will provide us with more ideas in this direction.

\section{A. Auxiliary results}

Here, we prove two technical results. The formulation of Lemma 4 could be simplified but in its present form its application in the proof of Lemma 5 is apparent.

Lemma 4. Given a three-space $E \subset P^{7}$ and four points $\left[u_{1}^{\prime}\right],\left[v_{1}^{\prime}\right],\left[u_{2}^{\prime}\right],\left[v_{2}^{\prime}\right]$ that span a three-space $F \subset P^{7}$ with $E \cap F=\varnothing$, set $U_{1}:=\left[u_{1}^{\prime}\right] \vee E, V_{1}:=\left[v_{1}^{\prime}\right] \vee E, U_{2}:=\left[u_{2}^{\prime}\right] \vee E$, $V_{2}:=\left[v_{2}^{\prime}\right] \vee E$ and consider four projections

$$
\mu_{1}: U_{1} \rightarrow V_{1}, \quad \nu_{1}: V_{1} \rightarrow U_{2}, \quad \mu_{2}: U_{2} \rightarrow V_{2}, \quad \nu_{2}: V_{2} \rightarrow U_{1}
$$

with respective centres $\left[m_{1}\right],\left[n_{1}\right],\left[m_{2}\right]$, and $\left[n_{2}\right]$. If the centres span a plane $L$, the composition $\kappa:=\nu_{2} \circ \mu_{2} \circ \nu_{1} \circ \mu_{1}$ of the four projections is the identity.

Proof. Note that $\operatorname{dim} U_{1} \vee V_{1}=5$ so that the projection $\mu_{1}$ (and also $\nu_{1}, \mu_{2}, \nu_{2}$ ) from a suitable point is well defined. Take an arbitrary point $\left[u_{1}\right] \in U_{1}$ but not in $E$ and not in $L$ and set $\left[v_{1}\right]:=\mu_{1}\left(\left[u_{1}\right]\right),\left[u_{2}\right]:=\nu_{1}\left(\left[v_{2}\right]\right),\left[v_{2}\right]:=\mu_{2}\left(\left[u_{2}\right]\right)$. Because these points are not coplanar, we may take them, in above order, as base points of a projective coordinate system in $G:=\left[u_{1}\right] \vee\left[v_{1}\right] \vee\left[u_{2}\right] \vee\left[v_{2}\right]$ and complement them by four further base points in $E$ and a suitable unit point to a projective coordinate system of $P^{7}$. We select the unit point such that its projection into $G$ from $E$ gives $\left(\left[m_{1}\right] \vee\left[m_{2}\right]\right) \cap\left(\left[n_{1}\right] \vee\left[n_{2}\right]\right)$ and denote induced projective coordinates in $U_{1}, V_{1}, U_{2}, V_{2}$, and $G$ (obtained by dropping three 
or four zero coordinates) by writing the subspace as subscript. With this convention, the projection centres are $\left[m_{1}\right]=[1,1,0,0]_{G},\left[n_{1}\right]=[0,1,1,0]_{G},\left[m_{1}\right]=[0,0,1,1]_{G}$, $\left[n_{2}\right]=[1,0,0,1]_{G}$. Moreover, we denote the coordinate vector of an arbitrary point $x \in U_{1}$ by $\left[x_{0}, \star\right]_{U_{1}}$ where the star abbreviates four fixed coordinates. We then have

$$
\left[x_{0}, \star\right]_{U_{1}} \stackrel{\mu_{1}}{\mapsto}\left[-x_{0}, \star\right]_{V_{1}} \stackrel{\nu_{1}}{\mapsto}\left[x_{0}, \star\right]_{U_{2}} \stackrel{\mu_{2}}{\mapsto}\left[-x_{0}, \star\right]_{V_{2}} \stackrel{\nu_{2}}{\mapsto}\left[x_{0}, \star\right]_{U_{1}}
$$

and the claim follows.

Lemma 5. Let $E, F \subset P^{7}$ be non-intersecting three spaces, the latter spanned by points $\left[u_{1}^{\prime}\right],\left[v_{1}^{\prime}\right],\left[u_{2}^{\prime}\right],\left[v_{2}^{\prime}\right]$. Consider further a regular quadric $\mathcal{Q}$ containing $E$ and four vertices $\left[m_{1}\right],\left[n_{1}\right],\left[m_{2}\right],\left[n_{2}\right] \in \mathcal{Q}$ of a planar quadrilateral. Then there exists a unique spatial quadrilateral with vertices $\left[u_{1}\right],\left[v_{1}\right],\left[u_{2}\right],\left[v_{2}\right]$ that is contained in $Q$, whose sides $\left[u_{1}\right] \vee\left[v_{1}\right],\left[v_{1}\right] \vee\left[u_{2}\right],\left[u_{2}\right] \vee\left[v_{2}\right],\left[v_{2}\right] \vee\left[u_{1}\right]$ are, in that order, incident with $\left[m_{1}\right],\left[n_{1}\right]$, $\left[m_{2}\right],\left[n_{2}\right]$ and whose projections from $E$ into $F$ are $\left[u_{1}^{\prime}\right],\left[v_{1}^{\prime}\right],\left[u_{2}^{\prime}\right]$, and $\left[v_{2}^{\prime}\right]$.

Proof. Denote a quadratic form associated to $\mathcal{Q}$ by $\omega$. Given $\left[u_{1}^{\prime}\right],\left[v_{1}^{\prime}\right],\left[u_{2}^{\prime}\right],\left[v_{2}^{\prime}\right]$, we have to reconstruct $\left[u_{1}\right],\left[v_{1}\right],\left[u_{2}\right],\left[v_{2}\right]$ subject to the constraints

$$
\omega\left(u_{i}, u_{i}\right)=\omega\left(v_{i}, v_{i}\right)=\omega\left(u_{i}, v_{j}\right)=0, \quad i, j \in\{1,2\}
$$

and

$$
\left[m_{1}\right] \in\left[u_{1}\right] \vee\left[v_{1}\right], \quad\left[n_{1}\right] \in\left[v_{1}\right] \vee\left[u_{2}\right], \quad\left[m_{2}\right] \in\left[u_{2}\right] \vee\left[v_{2}\right], \quad\left[n_{2}\right] \in\left[v_{2}\right] \vee\left[u_{1}\right] .
$$

If $\left[u_{1}\right]$ is given, we find $\left[v_{1}\right]$ by projecting $\left[u_{1}\right]$ from centre $\left[m_{1}\right]$ onto $E \vee\left[v_{1}^{\prime}\right]$. Lemma 4 tells us that we can find $\left[u_{2}\right]$ and $\left[v_{2}\right]$ in similar manner such that (4) is satisfied. Then, some of the conditions in (3) become redundant and it is sufficient to consider only

$$
\omega\left(u_{1}, u_{1}\right)=\omega\left(v_{1}, v_{1}\right)=\omega\left(u_{2}, u_{2}\right)=\omega\left(v_{2}, v_{2}\right)=0 .
$$

In a projective coordinate system with base points $\left[u_{1}^{\prime}\right],\left[v_{1}^{\prime}\right],\left[u_{2}^{\prime}\right],\left[v_{2}^{\prime}\right] \in F$ and further base points in $E$ the quadratic form $\omega$ (and the quadric $\mathcal{Q}$ ) is described by a matrix of the shape

$$
\left[\begin{array}{ll}
A & B \\
B & O
\end{array}\right]
$$

where $A, B$ and $O$ are matrices of dimension $4 \times 4, O$ is the zero matrix and $B$ is regular. Now (5) gives rise to a linear system for the unknown coordinates of $\left[u_{1}\right],\left[v_{1}\right],\left[u_{2}\right]$, and $\left[v_{2}\right]$. The matrix of the linear system is equivalent to the matrix $B$ by elementary row transformations, whence existence and uniqueness of a solution hinges solely on the regularity of $B$.

\section{Acknowledgement}

This work was supported by the Austrian Science Fund (FWF): P 26607 (Algebraic Methods in Kinematics: Motion Factorisation and Bond Theory). 


\section{References}

Brunnthaler, K., Schröcker, H.P. \& Husty, M., 2005 A new method for the synthesis of Bennett mechanisms. In Proceedings of CK 2005, International Workshop on Computational Kinematics, Cassino.

Cayley, A., 1885 On the twisted cubics upon a quadric surface. Messanger of Mathematics 14, 129-132.

Hamann, M., 2011 Line-symmetric motions with respect to reguli. Mech. Mach. Theory 46(7), 960-974.

Hegedüs, G., Schicho, J. \& Schröcker, H.P., 2013a Factorization of rational curves in the Study quadric and revolute linkages. Mech. Mach. Theory 69(1), 142152 .

Hegedüs, G., Schicho, J. \& Schröcker, H.P., 2013b The theory of bonds: A new method for the analysis of linkages. Mech. Mach. Theory 70, 407-424.

Hegedüs, G., Schicho, J. \& Schröcker, H.P., 2014 Four-pose synthesis of anglesymmetric 6R linkages. Accepted for publication in ASME J. Mechanisms Robotics.

Klawitter, D., 2015 Clifford Algebras. Geometric Modelling and Chain Geometries with Application in Kinematics. Springer Spektrum.

Salmon, G., 1882 A treatise on the analytic geometry of three dimensions. Hodges, Figgis \& Co., Dublin, 4 edn.

SELIG, J., 2005 Geometric Fundamentals of Robotics. Monographs in Computer Science, Springer, 2 edn.

SuH, C.H., 1969 On the duality in the existence of R-R links for three positions. ASME J. Mechanical Design 91(1), 129-134. 